\title{
Facile synthesis of highly processable and water dispersible polypyrrole and poly(3,4- ethylenedioxythiophene) microspheres for enhanced supercapacitive performance
}

Yu Liu, Anthony Turner, Maojun Zhao and Wing Cheung Mak

The self-archived postprint version of this journal article is available at Linköping University Institutional Repository (DiVA):

http://urn.kb.se/resolve?urn=urn:nbn:se:liu:diva-147165

N.B.: When citing this work, cite the original publication.

Liu, Yu, Turner, A., Zhao, M., Mak, W. C., (2018), Facile synthesis of highly processable and water dispersible polypyrrole and poly(3,4-ethylenedioxythiophene) microspheres for enhanced supercapacitive performance, European Polymer Journal, 99, 332-339.

https://doi.org/10.1016/j.eurpolymj.2017.12.013

Original publication available at:

https://doi.org/10.1016/j.eurpolymj.2017.12.013

Copyright: Elsevier

http://www.elsevier.com/

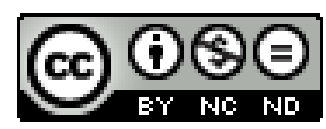




\title{
Facile synthesis of highly processable and water dispersible polypyrrole and poly(3,4-ethylenedioxythiophene) microspheres for enhanced supercapacitive performance
}

\author{
Yu Liu ${ }^{a, b}$, Anthony P.F. Turner ${ }^{a}$, Maojun Zhao ${ }^{b}$, Wing Cheung Mak ${ }^{a *}$ \\ a. Biosensors and Bioelectronics Centre, Department of Physics, Chemistry and Biology, Linköping University, SE-581 83 \\ Linköping, Sweden \\ b. College of Science, Sichuan Agricultural University, Yaan 625014, China \\ *corresponding author, E-mail: wing.cheung.mak@liu.se \\ Much recent work has focused on improving the processibility and electrocapacitive performance of conducting polymer-based materials for energy related \\ applications. The key mechanism of conducting polymers as supercapacitor materials as is driven by the rapid charging and discharging processes that involve \\ mass transport of the counter ions insertion/ejection within the polymer structure, where ion diffusion is usually the limiting step on the efficiency of the \\ conducting polymer capacitor. Here, we report a facile method for the green fabrication of polypyrrole microspheres (PPy-MSs) and poly (3, 4- \\ ethylenedioxythiophene) microspheres (PEDOT-MSs) with good processability, intact morphology and large active surface for enhanced ion interchange \\ processes, without using surfactant and highly irritant or toxic organic solvents during the synthetic process. The structure and morphology of the PPy-MSs \\ and PEDOT-MSs were characterized by means of SEM, EDX, TEM and FTIR. Both PPy-MSs and PEDOT-MSs showed intact microsphere structures with greatly \\ improved water dispersity and processability. More importantly, facilated by the large active surface and inter-microsphere space for ions diffusion, both the \\ PPy-MSs and PEDOT-MSs showed a signiciantly enhanced electrical capacitive performance of $242 \mathrm{~F} \mathrm{~g}^{-1}$ and $91.2 \mathrm{~F} \mathrm{~g}^{-1}$, repsectively (i.e. 10 and 1.51 times in \\ specific capactiance than the randomly structured PPy and PEDOT). This innovative approach not only addresses fundamental issues in fabrication of high \\ performance processable microstructured conducting polymers, but also makes progress in delivering water processable conducting polymers that could be \\ potentially used for fabrication of printed electronic devices.
}

Keywords: energy materials, conducting polymers, microspheres interface, colloidal chemistry

\section{Introduction}

Intrinsically conductive polymers (ICP), a class of organic polymers that conduct electricity, were first reported by Hideki et al. in 1977 [1]. As one of the most fascinating functional/conducting materials, organic conducting polymers have attracted considerable attention in recent years due to their unique electrical and physicochemical properties, such as electrical conductivity, high biocompatibility, good chemical stability, high electron affinity, low ionization potential and low energy optical transitions [2-9]. Therefore, conducting polymers are widely use in solar cells [10,11], supercapacitors [12-16], drug delivery vehicles [17-19], transparent conductive electrodes [20,21], organic semiconductor [22] and sensors $[\mathbf{2 3}, \mathbf{2 4}]$. However, their performance in such applications are profoundly affected by the structural morphology of the conducting polymer. An appropriate micro/nanostructure could improve the material processability and increase the effective surface area for the ion insertion processes, and thus enhance the performance of conducting polymer devices, such as printed biosensors [25] and supercapacitor electrodes [26]. Various kinds of conducting polymer micro/nanostructures have been developed, such as microspheres [27-33], nanofibers $[34,35]$, nanotubes [36], nanorods [37,38], nanowires [39], micro/nanoparticles[40,41] and microcapsules [42-44]. Among these micro/nanostructures, microspheres/particles have attracted special attention because of their spherical geometry, which minimizes surface energy thus reducing the aggregation effect, homogeneous distribution, and leads to easy processing into a colloidal suspension. In addition, microsphere particles are ideal building blocks for the construction of 2D or 3D devices incorporating hierarchical structured and processable materials for printed electronics [45].

Recently, many groups have reported the fabrication of particles based on polyaniline (PANI) and polypyrrole (PPy), while there are only few examples on poly $(3,4$-ethylenedioxythiophene) (PEDOT). This is due to the poor intrinsic properties of EDOT, such as a relatively low efficiency for oxidative polymerization and relatively low solubility of the EDOT monomer in the absence of surfactants $[\mathbf{4 2}, \mathbf{4 6}, \mathbf{4 7}]$. In addition, although the PEDOT:PSS solves the solubility problem of PEDOT[3,48], the good water solubility limits the diversity of polymer morphologies, and the doping of PSS also partly hinders the connection of the PEDOT chains which could weaken the conductivity [11]. Pud et al. synthesized a very small core-shell nanocomposite of polystyrene (PS) with doped PANI [49]. Jang et al. demonstrated the fabrication of silica-PANI core-shell nanoparticles [43]. Han and Foulger described the fabrication of nanometre-sized PEDOT-coated silica core-shell 
particles and PEDOT hollow particles [27]. Jiang et al. prepared porous PPy by using a nanoscale inorganic template [50]. Zhang and Suslick produced PEDOT microparticles by ultrasonic spray polymerization (USPo) [51]. Sui et al. synthesized PEDOT hollow spheres using surfactant mixtures as structure-directing agents to synthesize conducting polymer micro hollow spheres [33]. However, the fabrication of conducting polymer silica-core shell hybrid particles or surfactant directed methods with insulating doping materials could affect the overall conductivity and electrochemical performance of the resulting electrodes.

Moreover, most of the current methods have not avoided using some irritant reagents or toxic organic solvents that would be harmful to human health and the environment, such as hydrofluoric acid (HF) [27,52], 4-toluenesulfonic acid (TSA) [42], cetyltrimethylammonium bromide (CTAB) [33,38], ethylene oxide (EO) [53], tetrahydrofuran (THF) [54,55], and $\mathrm{N}, \mathrm{N}$ dimethyl formamide(DMF) [56]. Due to the fact that polymer is a soft material and as a consequence of solvent evaporation, the microparticles prepared by these methods usually suffer from shell collapse problems, which results in aggregation of microspheres in both the dried state and in common solvents. The instability in the microstructure could influence the efficiency of mass transport and the ion insertion process. The shell collapse is also a major drawback as building materials for fabrication of higher order 2D or 3D structures for real device applications. To the best of our knowledge, there is no compatible method for preparation of PPy and PEDOT microspheres. Thus, there is an urgent need to develop a green, simple and convenient method to synthesize conducting polymer microspheres with good processiblity, structural integrity and good electrocapacitive performance.

In this work, we describe such a green and robust method for synthesis of both PEDOT-MSs and PPy-MSs, which were obtained by employing $\mathrm{CaCO}_{3}$ as a common hard template for loading and polymerization of conducting polymer monomer, followed by a simple template removal using EDTA. The mechanism of synthesis of conducting polymer microspheres is shown in Figure 1. The fabrication method is surfactant-free and generally environmentally friendly (it only uses a small quantity of ethanol and isopropanol) and performed in aqueous media at room temperature. By this method, we overcame the structural instability issue, and introduced a new concept in the synthesis: mild oxidation followed by advanced oxidation for the fabrication of structural stable and intact microspheres. During the mild oxidation step, a primary framework of conducting polymer is formed, which is accompanied by a slow out-diffusion of the monomers from the $\mathrm{CaCO}_{3}$ template that happened simultaneously leading to insufficient polymer density within the microspheres. This was followed by an advanced oxidation step, to replenish new monomers in the template and allow further polymerization to reinforce the preexist polymer network and form a stable microsphere structure. The structural and chemical characteristics of the PPy-MSs and PEDOT-MSs obtained were examined by scanning electron microscopy (SEM), transmission electron microscopy (TEM), EDX, particle size and zeta potential analysis, and FTIR. We

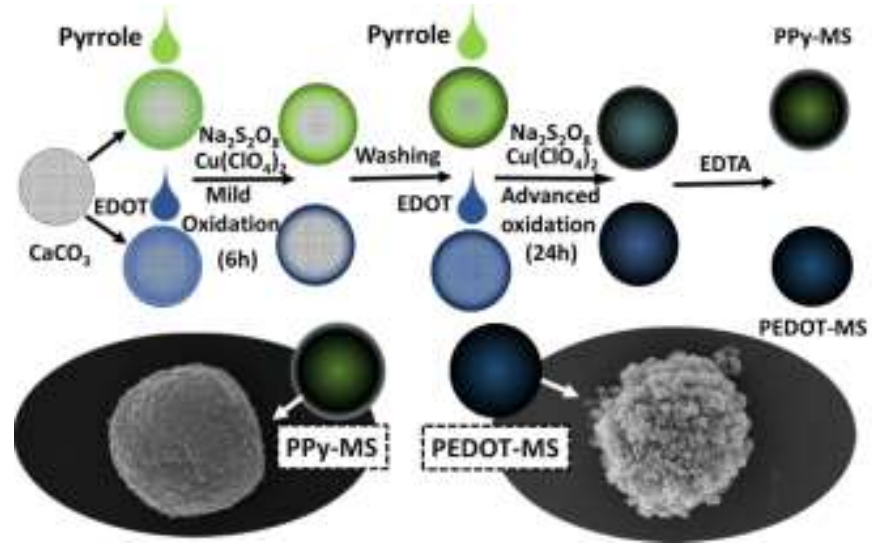

Figure 1 Schematic illustration of the formation mechanism of conducting polymer microspheres
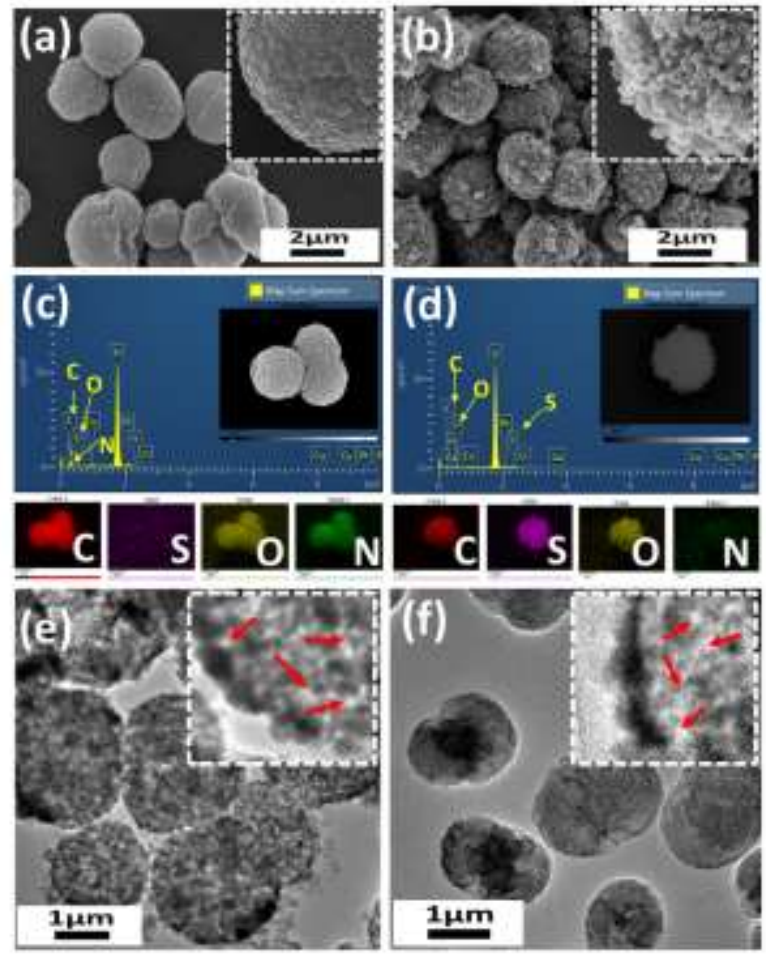

Figure 2 SEM images of (a) PPy-MSs, (b) PEDOT-MSs. EDS maps of (c) PPyMSs, (d) PEDOT-MSs. TEM images of (e) PEDOT-MSs and (f) PPy-MSs.

further examined the electrochemical capacitive properties of the PPy-MSs and PEDOT-MSs film with voltammetry and galvanostatic charge-discharge techniques.

\section{Results and discussion}

The morphology of the PPy-MSs and PEDOT-MSs were studied. Figure $\mathbf{2} \mathbf{a}$ and $\mathbf{2} \mathbf{b}$ show the SEM images of the PPy-MSs and PEDOT-MSs prepared by the two-step oxidation method using the $\mathrm{CaCO}_{3}$ template. It can be clearly seen that the PPy-MSs and PEDOT-MSs display intact spherical morphology without significant collapsed structures and maintaining a fairly uniform particle size (Supporting Information Figure S1). Their intact 
microsphere structure with good dispersibility in water might avail building block materials for further practical application and facilitate the ion diffusion or mass transport, which can enhance the electrochemical capacitive performance of these materials. These results demonstrated several advantages of the $\mathrm{CaCO}_{3}$ template-assisted synthesis approach. The inorganic hard template provides a relatively stable skeleton during the synthesis that allows easy manipulation of the multiple step process (two-stage oxidation processes), which is difficult to handle in conventional emulsification methods. The size and morphology of the resulting conducting polymer microspheres is governed by the $\mathrm{CaCO}_{3}$ template. The inorganic nature of the $\mathrm{CaCO}_{3}$ also allows the loading of the monomers with poor solubility, providing a compatible method for the fabrication of different conducting polymer microspheres. Moreover, the high magnification images show that the surface morphology of the PPy-MSs was smoother and compact compared with the PEDOT-MSs, which was rougher and looser (inset of Figure 2a and $\mathbf{2 b}$ ). This may due to the limited intrinsic properties of EDOT, such as a relatively low efficiency for oxidative polymerization leading to the formation of PEDOT-MSs with a relatively less dense polymer network with looser structure $[42,47]$. In addition, the same field of view was then examined by EDS (Figure 2c and Figure 2d). According to their own chemical structure, nitrogen is a characteristic element of pyrrole and sulphur for EDOT. From the EDS map, it is clear that
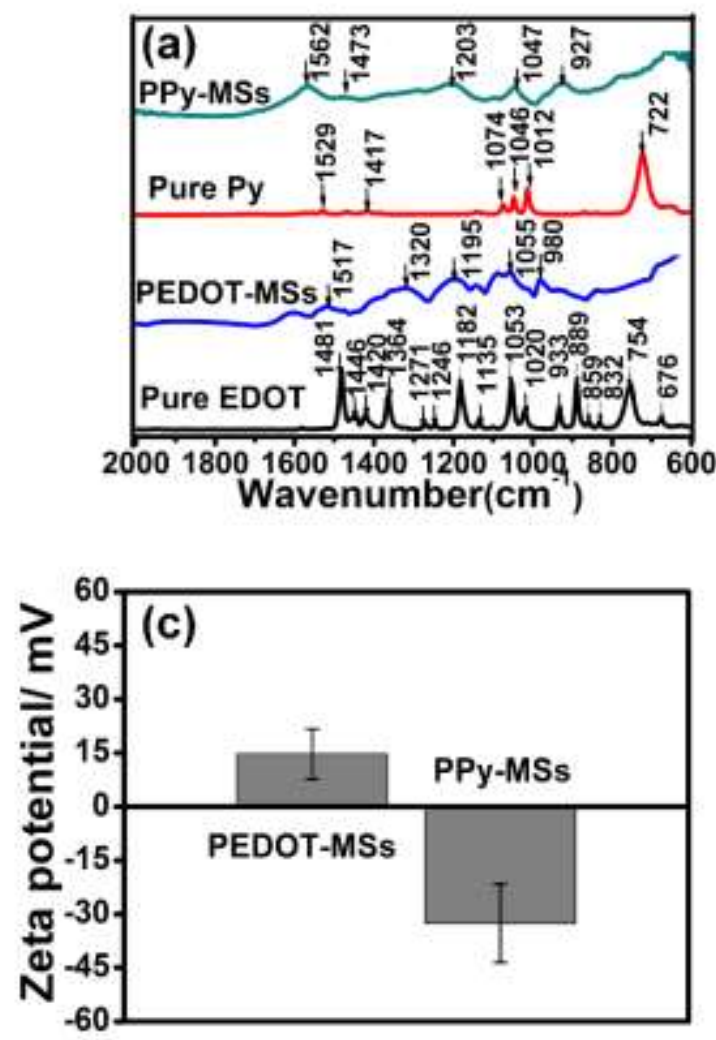

abundant nitrogen appears in the PPy-MSs, but there was a lack of sulphur. In contrast, PEDOT-MSs revealed a richness in sulphur, but an absence of nitrogen. In addition, there is no detectable calcium element in both PPy-MSs and PEDOT-MSs, which indicates the $\mathrm{CaCO}_{3}$ removal is very effective. The inner structure of the PEDOT-MSs and PPy-MSs were further characterized by TEM. As shown in Figure 2e, the PEDOT-MSs displayed an intact solid core microsphere structure. The uniform distribution of the bright and dark region are shown in high magnification TEM images suggest that PEDOT-MSs possess sponge-like porous structure (insert of Figure $2 \mathrm{e}$, arrows indicate the pores region). On the other hand, the TEM image of PPy-MSs (Figure 2f) appeared more homogeneous and lighter compared with the PEDOT-MSs, which indicates a higher porous internal structure (insert of Figure $2 f$, arrows indicate the pore region). Moreover, both PPy-MSs and PEDOT-MSs show a dense and homogenous internal structure, thus suggesting a relatively high polymerization efficiency of conducting polymer penetrating into the $\mathrm{CaCO}_{3}$ template leading to the formation of solid core particle structure. The EDS map of the TEM samples (Supporting Information Figure S2) is in agreement with the SEM-EDS showing that calcium was absent, which demonstrated the complete removal of $\mathrm{CaCO}_{3}$ template from within the internal structure of PPyMSs and PEDOT-MSs.
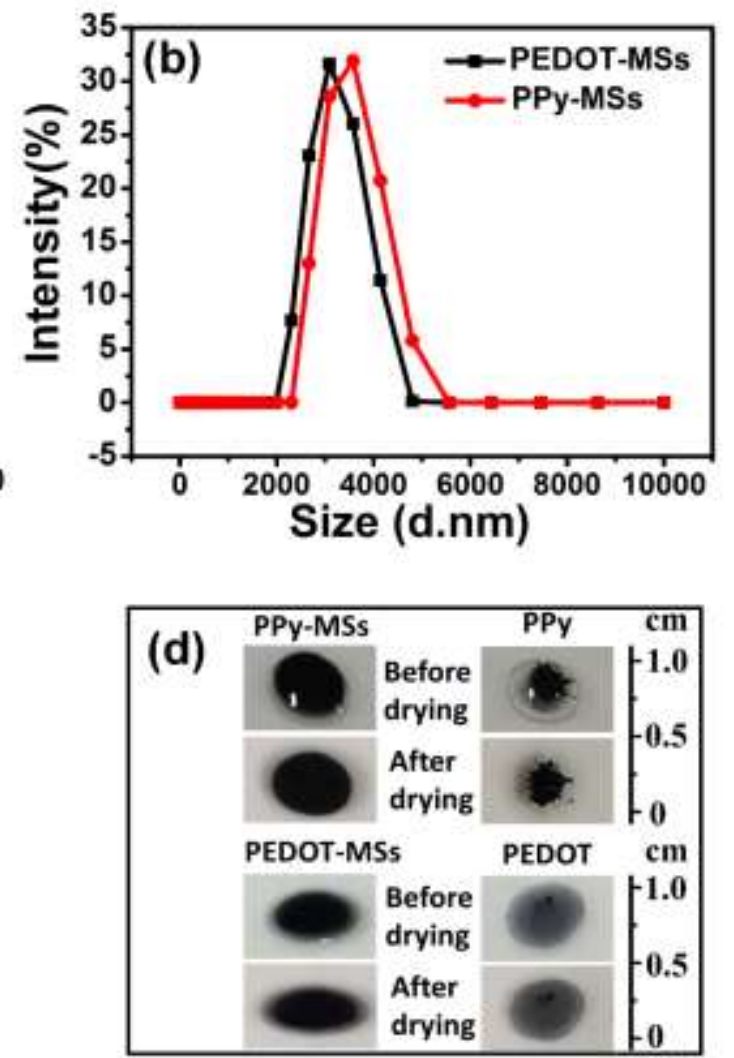

Figure 3 FTIR spectra of PPy-MSs, PEDOT-MSs, pure pyrrole and pure EDOT. (b) Size distribution curve of PPy-MSs and PEDOT-MSs. (c) Zeta potentials of PEDOT-MSs and PPy-MSs. (d) Optical photographs of dispersibility of PPy-MSs, randomly structured PPy, PEDOT-MSs and randomly structured PEDOT in water before drying and after drying. 
The FTIR spectrum of the synthesized PPy-MSs and PEDOTMSs, and the corresponding pure pyrrole and pure EDOT monomer are shown in Figure 3a. The characteristic bands of PPy-MSs observed in the spectrum at 1562 and $1473 \mathrm{~cm}^{-1}$, are assigned to the symmetric and asymmetric aromatic $\mathrm{C}=\mathrm{C}$ stretching vibrations, respectively. The absorption bands at about 1203 and $927 \mathrm{~cm}^{-1}$ are different characteristic bending modes of $\mathrm{C}-\mathrm{H}$ bending vibration. Meanwhile, the bands at 1203 and $927 \mathrm{~cm}^{-1}$ reflected the doping state of PPy-MSs [5,60-62]. While the band located at $1047 \mathrm{~cm}^{-1}$ is ascribed to the C-N stretching vibration. The absorption bands of PEDOT-MSs at 1320, 1195, 1055, $980 \mathrm{~cm}^{-1}$ originate from C-C, C=C, C-O-R-O-C and $\mathrm{C}-\mathrm{S}$ stretching. Moreover, the characteristic band at 754 $\mathrm{cm}^{-1}$, attributed to the $\mathrm{C}-\mathrm{H}$ out-of-plane bending vibration at $\mathrm{a}$ - $\alpha^{\prime}$ positions of EDOT, disappeared in the PEDOT-MSs, which demonstrated the EDOT polymerization was successful $[\mathbf{5 , 5 4 , 5 6 ]}$. All obtained spectra are in good agreement with those previously reported for pure pyrrole, EDOT and PPy, PEDOT in the literature. The FTIR results demonstrate the formation of PPy-MSs and PEDOT-MSs and their chemical structure obtained by chemical polymerization was not changed by the subsequent template removing process. What is more, the chemical polymerization reaction will produce PPy-MSs and PEDOT-MSs in an oxidized (conductive) form, which is important for creating a conductive structure for further applications. Figure $\mathbf{3 b}$ is the size distribution curve of PPy-MSs and PEDOT-MSs. It clearly shows a relatively narrow size distribution of PPy-MSs and PEDOT-MSs with mean diameters of $3.43 \pm 0.54$ and $3.18 \pm 0.52 \mu \mathrm{m}$, respectively. The surface charge of PPy-MSs, PEDOT-MSs were characterized by zeta potential measurements (Figure 3c). The PPy-MSs had a negative zeta potential value of $-32.6 \pm 11.0 \mathrm{mV}$ and the PEDOTMSs had a positive zeta potential value of $14.7 \pm 7.01 \mathrm{mV}$. Usually, PPy and PEDOT synthesized by chemical polymerization possess an oxidation state with dopant (i.e. $\mathrm{ClO}_{4}^{-}$) and carry a positive charge $[\mathbf{6 0}, \mathbf{4 8}, \mathbf{6 3}]$. While, here we observed that PPyMSs owned a negative charge at $\mathrm{pH}$ 7.4. This can be explained by the deprotonation of the nitrogen atoms of PPy under alkali conditions [63]. In our case, during the template removal process driven by the reaction between EDTA and $\mathrm{CaCO}_{3}$ resulting in the in-situ formation of $\mathrm{OH}^{-}$leading to the deprotonation of PPy, followed by the formation of negatively charged $\mathrm{PPy}-\mathrm{ClO}_{4} \mathrm{OH}^{-}$. This observation is further support by the EDS showing a strong oxygen characteristic peak for the PPyMSs (Figure 2e). Furthermore, the charged ICP microspheres indicate their doping state, which is the key factor for realizing higher capacitive performance of ICPs by a doping/de-doping process. During the redox reaction, the oxidation state of ICP electrode compels the dopants/counter ions and electrolyte to interchange through the internal porous structure of microspheres to maintain the electric neutrality of system. Meanwhile, the porous internal structure can facilitate the electrolyte ions to access the interior structure of the ICPS microspheres. Thus, both the porous structure and electrostatic forces can increase the ion exchange resulting in higher storage of electric charge. In order to intuitively observe the water dispersity of PPy-MSs and PEDOT-MSs, a droplet of the microspheres suspension was transferred onto a glass surface and dried, and the morphology of the resulting dry film was investigated (Figure 3d). The optical photographs clearly show that the PPy-MSs and PEDOT-MSs suspension in water media remains stable as a homogeneous black coloured solution, which is indicative of their good dispersity and MSs film appeared as a homogenously distributed coating layer. This indicates their potential application as processable coating materials for printed electronics. In contrast, the randomly structured PPy and PEDOT that were prepared in the absence of the $\mathrm{CaCO}_{3}$ template, had poor dispersity and significant aggregation, likely due to the high surface roughness of the random structure causing particle aggregation. More importantly, the particle aggregation would greatly restrict the ion diffusion efficiency and result in poor electrochemical performance.

The electrochemical behaviours of different conducting polymer films deposited onto glassy carbon electrode (GCE) were characterized by cyclic voltammetry (CV) and galvanostatic charging-discharging (GCD) techniques, to evaluate the influence on the structural morphology on the electrochemical performance of the PPy-MSs and PEDOT-MSs compared with the randomly structured PPy and PEDOT. All corresponding electrodes have the same mass of testing materials $(50 \mu \mathrm{g})$. The specific capacitance (Csp) from CV curves can be calculated according to: Eq. (1): Csp= $\int i \mathrm{dV} / \mathbf{m V ~ v}$

Where Csp is the specific capacitance, $i$ is the response current , $\mathrm{V}$ is the potential window, $\mathrm{v}$ is the potential scan rate, and $\mathrm{m}$ is the mass of the active material in the working electrode. The specific capacitance (Csp) from GCD curves can be calculated according to: Eq. (2): Csp=it/mV

Where $i$ is the charge-discharge current, $\mathrm{t}$ is the discharge time, $\mathrm{V}$ is the electrochemical window $(0.6 \mathrm{~V})$, and $\mathrm{m}$ is the mass of active material within the electrode. Figure $\mathbf{4 a}$ and $\mathbf{4 b}$ show the CV curves of PEDOT-MSs and PPy-MSs electrode in $0.5 \mathrm{M} \mathrm{H}_{2} \mathrm{SO}_{4}$ electrolyte in the potential range of -0.6 to $0.6 \mathrm{~V}$ at different scan rates from $10-500 \mathrm{mV} \mathrm{s}^{-1}$, respectively. The CV curves of PEDOT-MSs display a rectangular shape even at very high scan rates, indicating their excellent capacitive properties. For PPyMSs, the CV curve shows a sharp rise in the current in the low voltage region, followed by a significant drop at the vertex potential of $0.6 \mathrm{~V}$, and displaying good capacitive behaviour, especially at low scan rate. Figure $\mathbf{4 c}$ and $\mathbf{4 d}$ shows the representative GCD curves of PEDOT-MSs and PPy-MSs electrodes with the potential ranging from 0 to $0.6 \mathrm{~V}$ at different current densities from 0.4 to $1 \mathrm{~A} \mathrm{~g}^{-1}$. All curves display a triangular shape indicating their typical capacitive properties. The specific capacitances of the PPy-MSs, randomly structured PPy, PEDOT-MSs and randomly structured PEDOT electrodes calculated from the $\mathrm{CV}$ curves are plotted as a function of the scan rates in Figure $\mathbf{4 e}$. It can be seen that the specific capacitance of the PPy-MSs is higher than that of randomly structured PPy, especially at low scan rate. The maximum specific capacitance of the PPy-MSs is $194.7 \mathrm{~F} \mathrm{~g}^{-1}$, while the randomly structured PPy is only $24.2 \mathrm{~F} \mathrm{~g}^{-1}$ at a scan rate of 10 

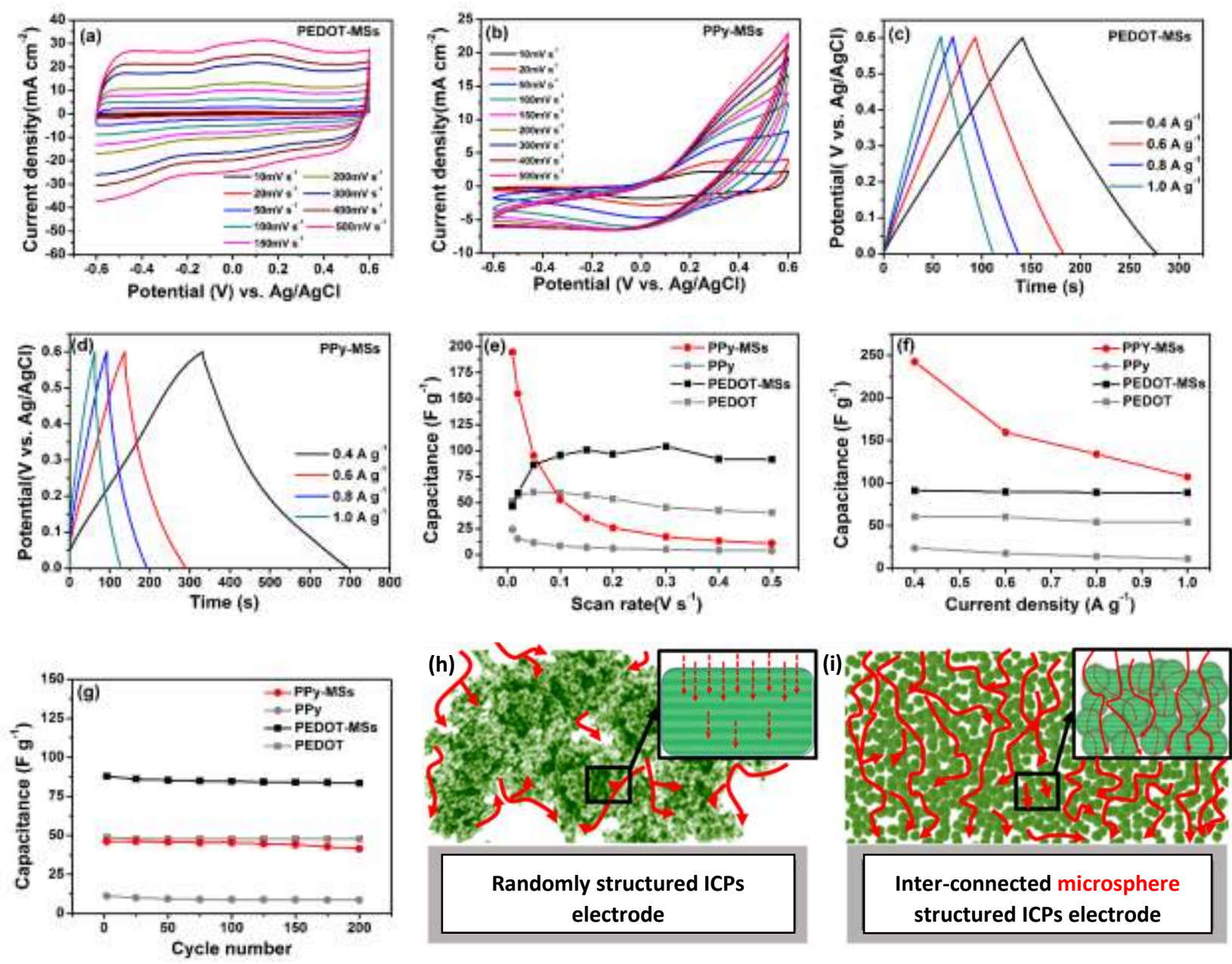

Figure 4 The CV curves of PEDOT-MSs (a) and PPy-MSs (b) at various scan rates. The CD curves of PEDOT-MSs (c) and PPy-MSs (d) at various current density from 0.4-1 A g-1. The specific capacitance as function of scan rate of different electrodes of PEDOT-MSs, PPy-MSs, random-structured PPy and PEDOT (e). The specific capacitances of PEDOT-MSs, PPy-MSs, randomly structured PPy and PEDOT electrodes at different current densities ( $f$ ). Stability of PEDOT-MSs, PPy-MSs, PEDOT and PPy electrodes with a relatively high scan rate of $100 \mathrm{mV} \mathrm{s}-1(\mathrm{~g})$. Schematic diagrams illustrate the proposed ion diffusion pathways in randomly structured ICPs (h) and inter-connected microsphere structured ICPs (i).

$\mathrm{mV} \mathrm{s}$. The term "maximum specific capacitance" here refers to the maximum value among tested points. The specific capacitance of the PPy-MSs increased by 2.8 to 10 times compared with the randomly structured PPy at different scan rates. Figure $\mathbf{4 e}$ also shows the specific capacitance of the PEDOT-MSs, which is higher than that of the randomly structured PEDOT. The specific capacitance of PEDOT-MSs increased almost $130 \%$ compared with the randomly structured PEDOT at a scan rate of $300 \mathrm{mV} \mathrm{s}^{-1}$, purely due to the morphological effect. The maximum specific capacitance of the PEDOT-MSs was $104.21 \mathrm{~F} \mathrm{~g}^{-1}$ and the randomly structured PEDOT was $59.97 \mathrm{~F} \mathrm{~g}^{-1}$ at a scan rate of $300 \mathrm{mV} \mathrm{s}^{-1}$ and $50 \mathrm{mV} \mathrm{s}$ 1 , respectively. The maximum difference in specific capacitance of the PEDOT-MSs and randomly structured PEDOT was 2.3 times with the value of $104.21 \mathrm{~F} \mathrm{~g}^{-1}$ and $45.27 \mathrm{~F} \mathrm{~g}^{-1}$ at a scan rate of $300 \mathrm{mV} \mathrm{s}^{-1}$. Figure $\mathbf{4 f}$ shows the specific capacitance calculated from the GCD curves of PPy-MSs, randomly structured PPy, PEDOT-MSs and randomly structured PEDOT electrodes under different current densities. It clearly shows that the specific capacitance of the PPy-MSs is much higher than that of the randomly structured PPy under all tested current densities. The PPy-MSs achieved a maximum specific capacitance of $242.6 \mathrm{~F} \mathrm{~g}^{-1}$ at a discharge current density of 0.4 A $\mathrm{g}^{-1}$, which is about 10 times as high compared with the randomly structured PPy of $23.8 \mathrm{~F} \mathrm{~g}^{-1}$ at $0.4 \mathrm{~A} \mathrm{~g}^{-1}$. The specific capacitance of PPy-MSs electrodes decreased significantly with increasing current density. This was probably caused by over oxidation of the conducting polymer at the higher current densities where it became unstable [6]. Nevertheless, the PPyMSs electrode shows the higher specific capacitance at low current density compared with the randomly structured PPy, suggesting that maintaining an intact microstructure and nonaggregated hierarchical organizational of the material is important for improved electrochemical capacitive performance. Similarly, the specific capacitance of the PEDOTMSs illustrated in Figure $\mathbf{4 f}$ is significantly higher than that of the randomly structured PEDOT under all the tested current densities. The PEDOT-MSs achieved a maximum specific capacitance of $91.2 \mathrm{~F} \mathrm{~g}^{-1}$ at a discharge current density of $0.4 \mathrm{~A}$ $\mathrm{g}^{-1}$, which is 1.51 times higher than the randomly structured 
PEDOT of $60.26 \mathrm{~F} \mathrm{~g}^{-1}$ at $0.4 \mathrm{~A} \mathrm{~g}^{-1}$. Furthermore, both the PEDOTMSs and randomly structured PEDOT electrodes retain a prominent rate capability of $97.6 \%$ and $89.7 \%$ at a high discharge current density of $1 \mathrm{~A} \mathrm{~g}^{-1}$, suggesting that PEDOT itself is a relatively more stable material compared with PPy for energy related applications. Figure $\mathbf{4 g}$ shows the cycling stability of the PPy-MSs, randomly structured PPy, PEDOT-MSs and randomly structured PEDOT electrodes measured at the scan rate of $100 \mathrm{mV} \mathrm{s}^{-1}$. The PPy-MSs retained $89.8 \%$ of the highest specific capacitance, while the randomly structured PPy retained only $77.3 \%$. The decrease may due to their structure being partly destroyed during the long process of ion insertion/ejection from the PPy chains during the measurement cycles. However, the PPy-MSs still shows a better stability compared with the randomly structured PPy. The spherical structural morphology may minimize the effect of film aggregation so as to reduce the resistance of ion diffusion and to maintain a higher electrochemical capacitive performance. The cycling stability of the PEDOT-MSs and randomly structured PEDOT electrodes retained $95 \%$ and $97.9 \%$ capacitance after 200 cycles, respectively. Both PEDOT-MSs and randomly structured PEDOT maintain a very stable performance. A possible reason for this is the good thermal and chemical stability of the 3, 4-disubstituted thiophene ring structure and fast electrochemical kinetics $[6,7,9,53]$. The good cycling stability may also likely be due to the relatively open intrinsic microstructure of the PEDOT-MSs, as shown in Figure $\mathbf{2} \mathbf{b}$, that facilitates the ion insertion/ejection processes even at a relative high scan rates and long cycling processes. All results demonstrate that the appropriate changes in the external microstructure of conducting polymers could enhance their electrochemical capacitive properties. As showed in Figure $\mathbf{4 h}$ and $\mathbf{4} \mathbf{i}$, the enhanced capacitive properties are possibly facilitated by the relatively homogenous micro-structured morphology of individual PPy-MSs and PEDOT-MSs and the space within the inter-microparticles network to promote ion diffusion and insertion process. In contrast, the serious aggregation of randomly structured PPy and PEDOT reduced the accessible surface area for ion diffusion and insertion into the ICPs.

\section{Conclusions}

We have demonstrated a compatible method for the bulk synthesis of highly dispersible and processable micro-structured PPy-MSs and PEDOT-MSs that does not require surfactants or highly toxic organic solvents. Both the PEDOT-MSs and PPy-MSs had an intact microsphere structure with fairly uniform diameter and good dispersity, and could be useful as building blocks for top-down fabrication of higher order 2D structured films. Both the PEDOT-MSs and PPy-MSs displayed significantly improved stability and electrochemical capacitive performance compared with randomly structured PPy and PEDOT, and demonstrated that the intact spherical morphology could minimize particle agglomeration, thus retaining the interparticle space that facilitates the ion diffusion/insertion into the microparticle film. The resulting processable conducting polymer microspheres could potentially be formulated into a functional ink for top-down large-scale fabrication of printed supercapacitors.

\section{Experimental}

Chemicals.

Calcium chloride $\left(\mathrm{CaCl}_{2}\right)$, sodium carbonate $\left(\mathrm{Na}_{2} \mathrm{CO}_{3}\right)$, copper (II) perchlorate hexahydrate $\mathrm{Cu}\left(\mathrm{ClO}_{4}\right)_{2} \cdot 6 \mathrm{H}_{2} \mathrm{O}$, sodium persulfate $\left(\mathrm{Na}_{2} \mathrm{~S}_{2} \mathrm{O}_{8}\right)$, ethylenediaminetetracetic acid (EDTA-2Na), ethanol, 2-propanol, 3, 4-ethylenedioxythiophene (EDOT) were purchased from Sigma-Aldrich (St. Louis, MO, USA) and were used as received. Pyrrole (99\%) (Py) was further purified before use by passing through a neutral column of alumina $(0.05 \mu \mathrm{m})$ to obtain a colourless liquid. All other chemicals were analytical grade and used without further purification. All solutions were prepared with milli-Q water.

\section{Preparation of porous calcium carbonate microparticles.}

Calcium carbonate microparticles were synthesized by a previously reported method with minor modifications [57-59]. Briefly, $2 \mathrm{~mL}$ of $1 \mathrm{M} \mathrm{Na}_{2} \mathrm{CO}_{3}$ solution was mixed with an equal volume of $1 \mathrm{M} \mathrm{CaCl}_{2}$ solution in a small beaker with stirring at 600 r.p.m. for $1 \mathrm{~min}$. The solution mixture turned opaque almost instantly. Then, the $\mathrm{CaCO}_{3}$ microparticles were washed by repeated centrifugation ( $130 \mathrm{~g}, 1 \mathrm{~min}$ ) and redispersed in milli-Q water and ethanol twice, followed by washing with 2propanol. The $\mathrm{CaCO}_{3}$ microparticles obtained were stored in 2propanol for further use.

\section{Preparation of PPy and PEDOT microspheres.}

A typical preparation process consisted of two key steps: mild oxidation and advanced oxidation. For the first mild oxidation step: Excess Py and EDOT monomers were loaded into the porous $\mathrm{CaCO}_{3}$ microparticles by mixing, and incubated for 2 hours to allow infusion of the monomer into the pores of the $\mathrm{CaCO}_{3}$ microparticles. Then, the monomer-loaded $\mathrm{CaCO}_{3}$ microparticles were washed with 2-propanol by centrifugation (130 g, $1 \mathrm{~min})$. To initiate the polymerization of the monomers, $500 \mu \mathrm{L}$ of $\mathrm{Na}_{2} \mathrm{~S}_{2} \mathrm{O}_{8}(0.2 \mathrm{M})$ and $120 \mu \mathrm{L}$ of $\mathrm{Cu}\left(\mathrm{ClO}_{4}\right)_{2}(0.5 \mathrm{M})$ were added to the monomer-loaded $\mathrm{CaCO}_{3}$ microparticles. The mixture was incubated for 6 hours at room temperature $\left(22^{\circ} \mathrm{C}\right)$. The reaction mixture was collected and centrifuged at $130 \mathrm{~g}$ for $2 \mathrm{~min}$, and then the pellet was resuspended and washed twice in 2-propanol, ethanol, milli-Q water and 2-propanol. For the advanced oxidation step: the pre-matured microparticles were mixed again with enough corresponding monomers, and incubated for 4 hours to allow a deep infusion of the monomers through the partially polymerized network into the interior of the $\mathrm{CaCO}_{3}$ microparticles. Then, the microparticles were washed by centrifugation ( $130 \mathrm{~g}, 1 \mathrm{~min}$ ) with 2-propanol and the same amount of oxidant as in the first step was added followed by deep polymerization of 24 hours. Then, the microparticle mixture was washed by centrifugation (130 g, $1 \mathrm{~min}$ ) with 2- 
propanol, ethanol and milli-Q water. Subsequently, the $\mathrm{CaCO}_{3}$ template was removed by the addition of $1 \mathrm{~mL}$ EDTA solution (0.2 M) followed by an incubation for 1 hour at room temperature $\left(22^{\circ} \mathrm{C}\right)$. Excess EDTA was removed by washing with milli-Q water three times by centrifugation ( $130 \mathrm{~g}, 1 \mathrm{~min}$ ) and re-dispersion cycles. The pure PPy-MSs and PEDOT-MSs were dried in a vacuum chamber for 48 hours, then the PPy-MSs and PEDOT-MSs were re-dispersed in milli-Q water to a concentration of $10 \mathrm{mg} \mathrm{mL}^{-1}$ and stored at room temperature $\left(22^{\circ} \mathrm{C}\right)$ for further characterization. The fabrication of randomly structured PPy and PEDOT were obtained using similar chemical procedures without the $\mathrm{CaCO}_{3}$ template.

\section{Morphology and Characterization.}

Scanning electron microscopy (SEM) images of PPy-MSs and PEDOT-MSs were recorded with a LEO 155 Gemini (Zeiss, OR, USA). The same field of view was then scanned using an energydispersive X-ray spectrometer (EDS) to acquire a set of X-ray maps for $\mathrm{C}, \mathrm{S}, \mathrm{O}$ and $\mathrm{N}$. All microspheres samples suspended in Milli-Q water were applied on a flat silicon surface, airdried at ambient temperature, and coated with platinum. Transmission electron microscopy (TEM) images of PPy-MSs and PEDOT-MSs were recorded with a G2 Sprit/Biotwin (FEI-Technai, Hillsboro, OR, USA).

\section{Particle Size and Zeta Potential Analysis.}

The particle size and zeta potentials (surface charge) of the PPyMSs and PEDOT-MSs were measured using a Zetasizer Nano ZS90 (Malvern Instruments Ltd., Worcestershire, U.K.). Particle size was measured on the basis of the dynamic light scattering technique, measuring the Brownian motion of the particles and converting the data into a size distribution graph using the Stokes-Einstein relationship. The zeta potential was measured on the basis of the laser Doppler micro-electrophoresis principle. The electrophoretic mobility $(\mu)$ was converted to the

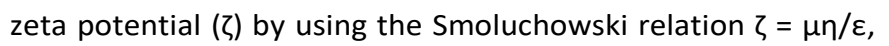
where $\eta$ and $\varepsilon$ are the viscosity and permittivity of the solution, respectively. One milliliter of suspended microspheres in water was loaded into a zeta potential measuring cell. The measurements were performed at $25{ }^{\circ} \mathrm{C}$, and the mean zeta potential values were calculated by taking an average of three repeated measurements.

\section{Fourier Transform Infrared Spectroscopy (FTIR).}

Fourier transform infrared (FTIR) spectroscopy was performed with VERTEX 70 (Bruker, USA) equipped with a germanium attenuated total reflectance (ATR) sample cell. Microsphere suspensions were dropped onto the surface of the ATR cell, and FTIR spectra were recorded in the frequency region of 600 to $2000 \mathrm{~cm}^{-1}$ with a resolution of $4 \mathrm{~cm}^{-1}$ and run for 100 cycles.

\section{Electrochemical Measurements.}

All electrochemical experiments and simulations were performed with a PGSTAT30 potentiostat (Autolab, Nertherland) under NOVA or GPES software control employing a conventional three-electrode electrochemical system. A silver chloride electrode $(\mathrm{Ag} / \mathrm{AgCl}, 3 \mathrm{M} \mathrm{KCl})$ and a Pt wire were used as the reference and counter electrode, respectively. A glassy carbon electrode (GCE, $3 \mathrm{~mm}$ diameter, surface area 0.0707 $\mathrm{cm}^{2}$ ) was used as the working electrode after being polished to mirror-like finish with $1.0,0.3$, and $0.05 \mu \mathrm{m}$ alumina slurries successively and washed ultrasonically in milli-Q water. $5 \mu \mathrm{L}$ of the conducting polymer ink ( $10 \mathrm{mg} \mathrm{mL}^{-1}$ ) was deposited onto the GCE and air dried overnight, followed by deposition of $2 \mu \mathrm{L}$ of 0.05 wt.\% Nafion ethanol solution for packaging and protection of the electrode surface. Finally, the electrochemical performance of the PPy-MSs and PEDOT-MSs electrodes were evaluated by cyclic voltammetry (CV) and galvanstatic chargedischarge (GCD). All current densities in the manuscript were calculated by normalizing the current with the absolute mass of PPy-MSs and PEDOT-MSs. All experiments were carried out at ambient temperature.

\section{Acknowledgements}

The authors would like to acknowledge the Swedish Re-search Council (VR-2015-04434) and China Scholarship Council (CSC) (File No. 201406910068) for generous financial support to carry out this research.

\section{References}

1 H. Shirakawa, E. J. Louis, A. G. MacDiarmid, C. K. Chiang and A. J. Heeger, Synthesis of Electrically Conducting Organic Polymers: Halogen Derivatives of Polyacetylene, $(\mathrm{CH})_{x}$, J.C.S. Chem. Comm. 16(1977) 578-580.

2 B. J. Feldman, P. Burgmayer, R. W. Murray, The Potential Dependence of Electrical Conductivity and Chemical Charge Storage of Poly(Pyrrole) Films on Electrodes, J. Am. Chem. Soc. 107(1985) 872-878

3 R. Gangopadhyay, B. Das, M. R. Molla, How Does PEDOT Combine with PSS? Insights from Structural Studies, RSC Adv. 4(2014) 43912-43920.

4 H. C. Kang, K. E. Geckeler, Enhanced Electrical Conductivity of Polypyrrole Prepared by Chemical Oxidative Polymerization: Effect of The Preparation Technique and Polymer Additive, Polymer 41(2000) 6931-6934.

5 A. S. Saraç, G. Sönmez, F.C. Cebeci, Electrochemical Synthesis and Structural Studies of Polypyrroles, Poly(3,4ethylenedioxythiophene)s and Copolymers of Pyrrole and 3,4Ethylenedioxythiophene on Carbon Fibre Microelectrodes, J. Appl. Electrochem. 33(2003) 295-301.

6 G. A. Snook, P. Kao, A. S. Best, Conducting-polymer-based Supercapacitor Devices and Electrodes, I Power Sources 196(2011) 1-12.

7 M. A. Khan, S. P. Armes, Synthesis and Characterization of Micrometer-Sized Poly(3,4-ethylenedioxythiophene)-Coated Polystyrene Latexes, Langmuir 15(1999) 3469-3475.

8 J. Rivnay, S. Inal, B. A. Collins, M. Sessolo, E. Stavrinidou, X. Strakosas, C. Tassone, D. M. Delongchamp, G. G. Malliaras, Structural Control of Mixed Ionic and Electronic Transport in Conducting Polymers, Nat. Commun. 7(2016) 11287.

9 S.-G. Oh and S.-S. Im, Electroconductive Polymer Nanoparticles Preparation and Characterization of PANI and PEDOT Nanoparticles, Curr. Appl. Phys., 2(2002) 273-277. 
10 Q. Xu, T. Song, W. Cui, Y. Liu, W. Xu, S. T. Lee, B. Sun, SolutionProcessed Highly Conductive PEDOT:PSS/AgNW/GO Transparent Film for Efficient Organic-Si Hybrid Solar Cells, ACS Appl. Mater. Inter. 7(2015) 3272-3279.

11 D. Alemu, H-Y. Wei, K-C Ho, C-W Chu, Highly conductive PEDOT:PSS electrode by simple film treatment with methanol for ITO-free polymer solar cells, Energ. Environ. Sci. 5 (2012) 9662-9671.

12 Z. Li, G. Ma, R. Ge, F. Qin, X. Dong, W. Meng, T. Liu, J. Tong, F. Jiang, Y. Zhou, K. Li, X. Min, K. Huo, Free-Standing Conducting Polymer Films for High-Performance Energy Devices, Angew. Chem. Int. Ed. Engl. 55(2016) 979-982.

13 W. Jiang, D. Yu, Q. Zhang, K. Goh, L. Wei, Y. Yong, R. Jiang, J. Wei, Y. Chen, Ternary Hybrids of Amorphous Nickel Hydroxide-Carbon Nanotube-Conducting Polymer for Supercapacitors with High Energy Density, Excellent Rate Capability, and Long Cycle Life, Adv. Funct. Mater. 25(2015) 1063-1073.

14 W. Fan, C. Zhang, W. W. Tjiu, K. P. Pramoda, C. He, T. Liu, Graphene-Wrapped Polyaniline Hollow Spheres as Novel Hybrid Electrode Materials for Supercapacitor Applications ACS Appl. Mater. inter. 5(2013) 3382-3391.

15 Y. Zeng, Y. Han, Y. Zhao, Y. Zeng, M. Yu, Y. Liu, H. Tang, Y. Tong, X. Lu, Advanced Ti-Doped Fe2O3@PEDOT Core/Shell Anode for High-Energy Asymmetric Supercapacitors. Adv. Energy Mater. 5 (2015) 1402176.

16 D. Yu, K. Goh, H. Wang, L. Wei, W. Jiang, Q. Zhang, L. Dai, Y. Chen, Scalable Synthesis of Hierarchically Structured Carbon Nanotube-Graphene Fibres for Capacitive Energy Storage, Nat. Nanotechnol. 9(2014) 555-562.

17 E. Shamaeli, N. Alizadeh, Studies of Electrochemically Controlled Release of Salicylate from Nanostructure Conducting Molecularly Imprinted Polymer, Electrochim. Acta 114(2013) 409-415.

18 G. Kaur, R. Adhikari, P. Cass, M. Bown, P. Gunatillake, Electrically Conductive Polymers and Composites for Biomedical Applications, RSC Adv.5(2015) 37553-37567.

19 S. Löffler, B. Libberton, A. Richter-Dahlfors, Organic Bioelectronic Tools for Biomedical Applications, Electronics 4(2015) 879-908.

20 B. Crone, A. Dodabalapur, Y. Y. Lin, R. W. Filas, Z. Bao, A. LaDuca, R. Sarpeshkar, H. E. Katz, W. Li, Large-Scale Complementary Integrated Circuits Based on Organic Transistors, Nature 403 (2000) 521-523.

21 Z. Liu, K. Parvez, R. Li, R. Dong, X. Feng, K. Mullen, Transparent Conductive Electrodes from Graphene/PEDOT:PSS Hybrid Inks for Ultrathin Organic Photodetectors, Adv. Mater. 27 (2015) 669-675.

22 H. E. Katz, A. J. Lovinger, J. Johnson, C. Kloc, T. Siegrist, W. Li, Y. Y. Lin, A. Dodabalapur, A Soluble and Air-Stable Organic Semiconductor with High Electron Mobility, Nature 404 (2000) 478-481.

23 F. S. Omar, N. Duraisamy, K. Ramesh, S. Ramesh, Conducting Polymer and Its Composite Materials Based Electrochemical Sensor for Nicotinamide Adenine Dinucleotide (NADH), Biosens. Bioelectron.79 (2016) 763-775.

24 Y. Liu, A.P.F. Turner, M. Zhao, W. C. Mak, Processable enzymehybrid conductive polymer composites for electrochemical biosensing, Biosensors and Bioelectronics 100 (2018) 374-381

25 R. Wannapob, M. Y. Vagin, Y. Liu, P. Thavarungku, P. Kanatharana, A. P. F. Turner, W. C. Mak, Printable Heterostructured Bioelectronic Interfaces with Enhanced Electrode Reaction Kinetics by Intermicroparticle Network. ACS Appl. Mater. Interfaces 9 (2017) 33368-33376

26 M. Y. Vagin, R. Wannapob, Y. Liu, W. C. Mak, Potentialmodulated electrocapacitive properties of soft microstructured polypyrrole, Electroanalysis, 29(2017) 203207

27 M. G. Han, S. H. Foulger, Preparation of Poly $(3,4$ ethylenedioxythiophene)(PEDOT) Coated Silica Core-Shell Particles and PEDOT Hollow Particles, Chem. Commun. 19 (2004)2154-2155.

28 P. Liu, X. Wang, Y. Wang, Design of Carbon Black/Polypyrrole Composite Hollow Nanospheres and Performance Evaluation as Electrode Materials for Supercapacitors, ACS Sustain. Chem. Eng. 2(2014) 1795-1801.

29 F. Caruso, R. A. Caruso, H. Mohwald, Nanoengineering of Inorganic and Hybrid Hollow Spheres by Colloidal Templating Science 282 (1998) 1111-1114.

30 Z. Guo, Y. Qiao, H. Liu, C. Ding, Y. Zhu, M. Wan, L. Jiang, SelfAssembled Hierarchical Micro/Nano-Structured PEDOT as An Efficient Oxygen Reduction Catalyst Over A Wide pH Range, J. Mater. Chem. 22 (2012) 17153.

31 T. L. Kelly, K. Yano, M. O. Wolf, Supercapacitive Properties of PEDOT and Carbon Coloidal Microspheres, ACS Appl. Mater. Inter. 1 (2009) 2536-2543.

32 X. Ni, X. Hu, S. Zhou, C. Sun, X. Bai, P. Chen, Synthesis and Microwave Absorbing Properties of Poly(3,4ethylenedioxythiophene) (PEDOT) Microspheres, Polym. Advan. Technol. 22(2011) 532-537.

33 J. Sui, L. Zhang, J. Travas-Sejdic, P. A. Kilmartin, Synthesis of Poly(3,4-ethylenedioxythiophene) Hollow Spheres in CTAB/DBS-Mixed Surfactant Solutions, Macromol. Symp. 290 (2010) $107-114$.

34 Y.-Z. Long, M.-M. Li, C. Gu, M. Wan, J.-L. Duvail, Z. Liu, Z. Fan, Recent Advances in Synthesis, Physical Properties and Applications of Conducting Polymer Nanotubes and Nanofibers, Prog. Polym. Sci. 36 (2011) 1415-1442.

35 N. Kim, S. Kee, S. H. Lee, B. H. Lee, Y. H. Kahng, Y. R. Jo, B. J. Kim, K. Lee, Highly Conductive PEDOT:PSS Nanofibrils Induced by Solution-Processed Crystallization, Adv. Mater. 26 (2014) 2268-2272.

36 R. Liu, S. I. Cho, S. B. Lee, Poly(3,4-ethylenedioxythiophene) Nanotubes As Electrode Materials for a High-Powered Supercapacitor, Nanotechnology 19 (2008) 215710.

37 O. Khani, F. Nemati, H. Farrokhi, M. Jazirehpour, Synthesis and characterization of electromagnetic properties of polypyrrole nanorods prepared via self-reactive $\mathrm{MnO} 2$ template Synthetic Met. 220 (2016) 567-572.

$38 \mathrm{Y}$. Li, B. Wang, H. Chen, W. Feng, Improvement of the Electrochemical Properties via Poly(3,4Ethylenedioxythiophene) Oriented Micro/Nanorods, J. Power Sources 195 (2010) 3025-3030.

39 Z. Yin, Q. Zheng, Controlled Synthesis and Energy Applications of One-Dimensional Conducting Polymer Nanostructures: An Overview, Adv. Energy Mater. 2 (2012) 179-218.

40 Oleg P. Dimitriev, Effect of Confinement on Photophysical Properties of P3HT Chains in PMMA Matrix, Nanoscale Res. Lett. 12 (2017) 510

41 G. M. Neelgund, V. N. Bliznyuk a, A. A. Pud, K. Y. Fatyeyeva, E. Hrehorova, M. Joyce, Formation of nanostructured composites with environmentally-dependent electrical properties based on poly(vinylidene fluoride)epolyaniline coreeshell latex system, Polymer 51 (2010) 2000-2006

42 M. G. Han, S. P. Armes, Synthesis of Poly(3,4ethylenedioxythiophene)/Silica Colloidal Nanocomposites, Langmuir 19 (2003) 4523-4526.

43 J. Jang, J. Ha, B. Lim, Synthesis and characterization of monodisperse silica-polyaniline core-shell nanoparticles, Chem. Commun. 15 (2006) 1622-1624.

44 N. A. Ogurtsov, Y. V. Noskov, K. Y. Fatyeyeva, V. G. Ilyin, G. V. Dudarenko, A. A. Pud, Deep Impact of The Template on 
Molecular Weight, Structure, and Oxidation State of The Formed Polyaniline, J. Phys. Chem. B 117 (2013) 5306-5314.

45 L. Pan, H. Qiu, C. Dou, Y. Li, L. Pu, J. Xu, Y. Shi, Conducting Polymer Nanostructures: Template Synthesis and Applications in Energy Storage, Int. J. Mol. Sci. 11 (2010) 26362657.

46 A. M. J. Henderson, J. M. Saunders, J. Mrkic, P. Kent, J. Gore, B. R. Saunders, A New Method for Stabilising Conducting Polymer Latices Using Short Chain Alcohol Ethoxylate Surfactants, J. Mater. Chem. 11 (2001) 3037-3042.

47 N. Paradee, A. Sirivat, Synthesis of Poly(3,4ethylenedioxythiophene) Nanoparticles via Chemical Oxidation Polymerization, Polym. Int. 63 (2014) 106-113.

48 O. P. Dimitriev, Y. P. Piryatinski, A. A. Pud, Evidence of The Controlled Interaction Between PEDOT and PSS In The PEDOT:PSS Complex via Concentration Changes of The Complex Solution, J. Phys. Chem. B 115 (2011) 1357-1362.

49 A. A. Pud, O. A. Nikolayeva, L. O. Vretik, Yu. V. Noskov, N. A. Ogurtsov, O. S. Kruglyak, E. A. Fedorenko, New nanocomposites of polystyrene with polyaniline doped with lauryl sulfuric acid, Nanoscale Res. Lett. 12 (2017) 493.

50 F. Jiang, T. Zhou, S. Tan, Y. Zhu, Y. Liu, D. Yuan, Porous Polypyrrole Prepared by Using Nanoscale Calcium Carbonate as a Core for Supercapacitance Materials, Int. J. Electrochem. Sci. 4 (2009) 1541-1547.

51 Y. Zhang, K. S. Suslick, Synthesis of Poly(3,4ethylenedioxythiophene) Microspheres by Ultrasonic Spray Polymerization (USPo), Chem. Mater. 27 (2015) 7559-7563.

52 T. L. Kelly, Y. Yamada, S. P. Y. Che, K. Yano, M. O. Wolf Monodisperse Poly(3,4 - ethylenedioxythiophene)-Silica Microspheres: Synthesis and Assembly into Crystalline Colloidal Arrays, Adv. Mater. 20 (2008) 2616-2621.

53 M. Mumtaz, A. de Cuendias, J.-L. Putaux, E. Cloutet, H. Cramail, Synthesis of PEDOT Nanoparticles and Vesicles by Dispersion Polymerization in Alcoholic Media, Macromol. Rapid. Comm. 27 (2006) 1446-1453.

54 Y. Zhang, M. Xin, W. Lin, Z. Yu, J. Peng, K. Xu, M. Chen, Synthesis and Characterization of PEDOT Particles in Aqueous
Dispersion of $\pi$-Conjugated Polyelectrolyte, Synthetic Met. 193 (2014) 8-16.

55 S. C. Luo, H. H. Yu, A. C. Wan, Y. Han, J. Y. Ying, A General Synthesis for PEDOT-Coated Nonconductive Materials and PEDOT Hollow Particles by Aqueous Chemical Polymerization, Small 4 (2008) 2051-2058.

56 J. Wu, Y. Li, W. Feng, A Novel Method to Form Hollow Spheres of Poly(3,4-ethylenedioxythiophene): Growth from A SelfAssemble Membrane Synthesized by Aqueous Chemical Polymerization, Synthetic Met. 157 (2007) 1013-1018.

57 D. V. Volodkin, A. I. Petrov, M. Prevot, G. B. Sukhorukov, Matrix Polyelectrolyte Microcapsules: New System for Macromolecule Encapsulation, Langmuir 20 (2004) 33983406.

58 D. V. Volodkin, R. von Klitzing, H. Mohwald, Pure Protein Microspheres by Calcium Carbonate Templating, Angew. Chem. Int. Ed. Engl. 49 (2010) 9258-9261.

59 G. B. Sukhorukov, D. V. Volodkin, A. M. Günther, A. I. Petrov, D. B. Shenoy, H. Möhwald, Porous Calcium Carbonate Microparticles as Templates for Encapsulation of Bioactive Compounds, J. Mater. Chem. 14 (2004) 2073-2081.

60 J. Lei, C. R. Martin, Infrared Investigations of Pristine Polypyrrole-Is The Polymer Called Polypyrrole Really Poly(pyrrole-co-hydroxypyrrole)?, Synthetic Met. 48 (1992) 331-336.

61 Y. Shi, L. Pan, B. Liu, Y. Wang, Y. Cui, Z. Bao, G. Yu Nanostructured Conductive Polypyrrole Hydrogels as HighPerformance, Flexible Supercapacitor Electrodes, J. Mater. Chem. A 2 (2014) 6086.

62 J. Xu, D. Wang, L. Fan, Y. Yuan, W. Wei, R. Liu, S. Gu, W. Xu Fabric Electrodes Coated with Polypyrrole Nanorods for Flexible Supercapacitor Application Prepared via A Reactive Self-Degraded Template, Org. Electron. 26 (2015) 292-299.

$63 \mathrm{X}$. Zhang, R. Bai, Surface Electric Properties of Polypyrrole in Aqueous Solutions, Langmuir 19 (2003) 10703-10709 
Supporting Information

Facile synthesis of highly processable and water dispersible polypyrrole and poly(3,4-ethylenedioxythiophene) microspheres for enhanced supercapacitive performance

Yu Liu ${ }^{a, b}$, Anthony P.F. Turner ${ }^{a}$, Maojun Zhao ${ }^{b}$, Wing Cheung Mak ${ }^{a *}$

aBiosensors and Bioelectronics Centre, Department of Physics, Chemistry and Biology, Linköping University, SE-581 83 Linköping, Sweden

${ }^{\mathrm{b} C o l l e g e ~ o f ~ S c i e n c e, ~ S i c h u a n ~ A g r i c u l t u r a l ~ U n i v e r s i t y, ~ Y a a n ~ 625014, ~ C h i n a ~}$
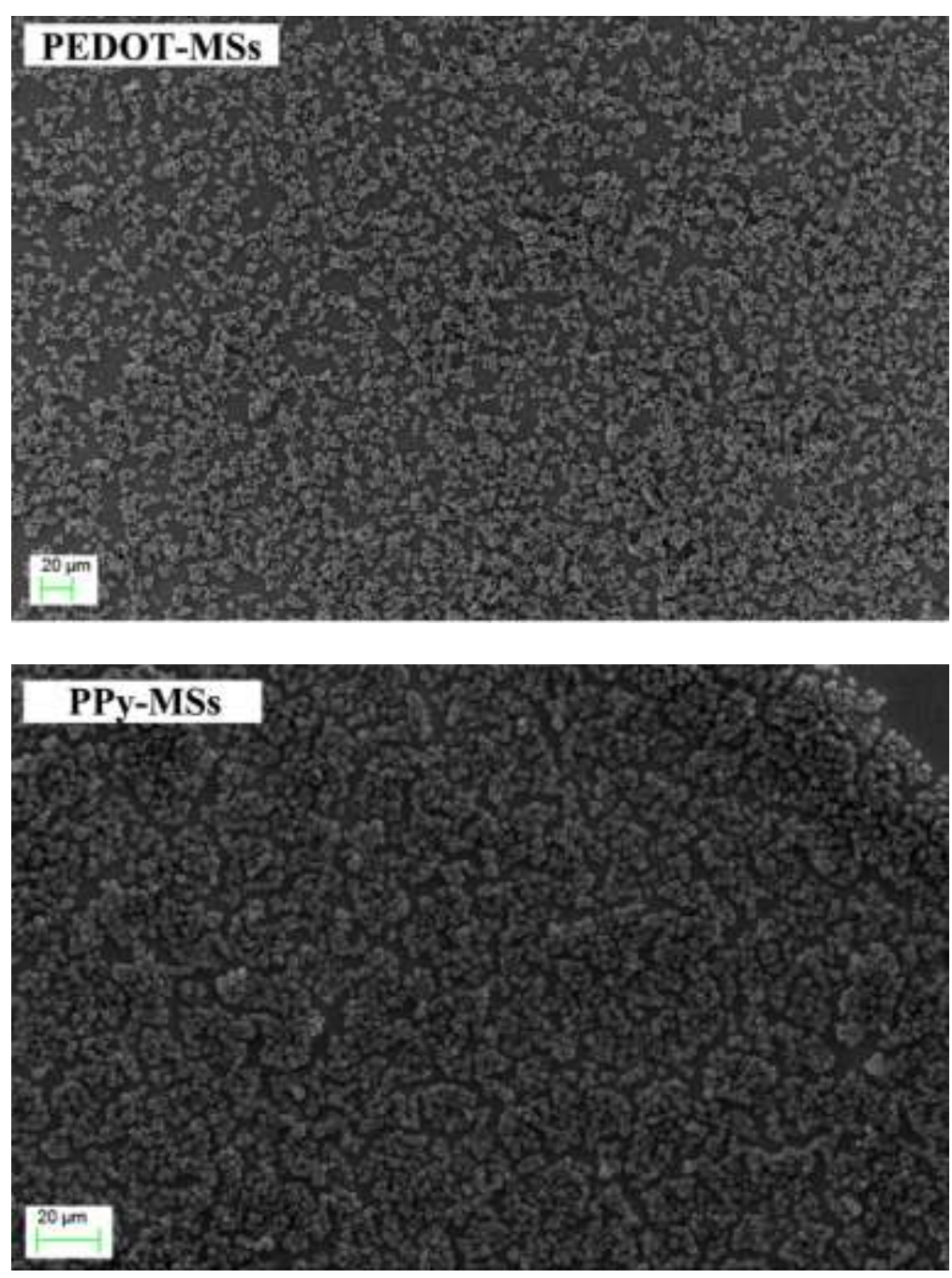

Figure S1. SEM images of PEDOT-MSs and PPy-MSs with at low magnifications. 

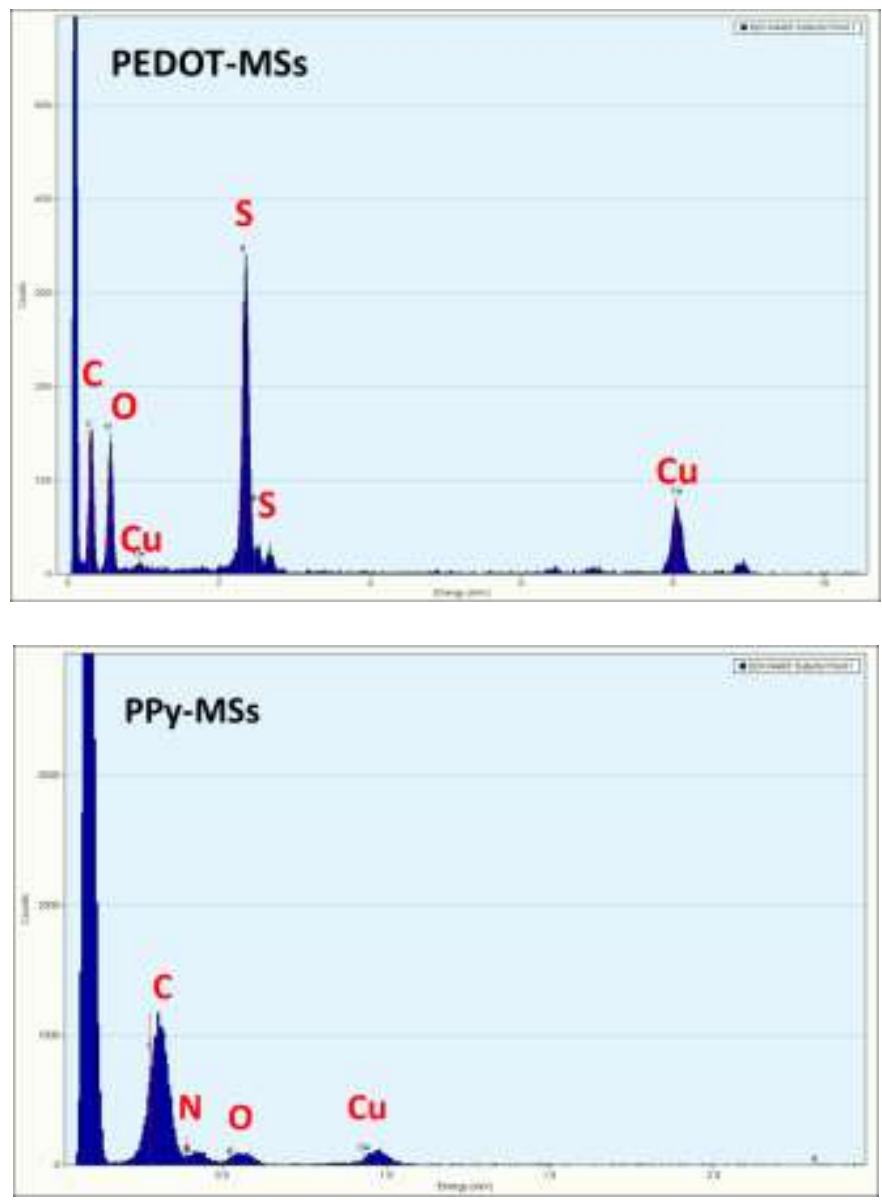

Figure S2. TEM-EDX spectrum of PEDOT-MSs and PPy-MSs 